\title{
Scientific Opportunities for FEL Amplifier based VUV and X-ray Research*
}

\author{
Erik D. Johnson \\ Brookhaven National Laboratory, National Synchrotron Light Source \\ Upton New York 11973-5000
}

\begin{abstract}
It has become increasingly clear to a wide cross section of the synchrotron radiation research community that FELs will be the cornerstone of Fourth Generation Radiation Sources. Through the coherent generation of radiation, they provide as much as 12 orders of magnitude increase in peak power over the third generation storage ring machines of today. Facilities have been proposed which will extend the operating wavelength of these devices well beyond the reach of existing solid state laser technology. In addition, it appears possible to generate pulses of unprecedented brevity, down to a few femtoseconds, with $\mathrm{mJ}$ pulse energies. The combination of these attributes has stimulated considerable interest in short wavelength FELsfor experiments in chemical, surface, and solid state physics, biology and materials science. This paper provides a brief overview of how the features of these FEL's relate to the experimental opportunities.
\end{abstract}

\section{Introduction:}

Although the subject of this paper has intentionally been limited to research applications utilizing short wavelength FEL amplifiers, the number of qualifying adjectives in the title is still insufficient to allow the comprehensive treatment of the subject in the available space. The discussion instead will highlight general features of these types of sources and how their attributes determine the choice of experiment and methodology for which they are appropriate. This selective treatment of short wavelength FEL science is not intended either to represent, nor minimize, the potential of oscillator based sources, or the growing body of industrial and technological applications for which FEL sources may be appropriate. They are simply beyond the scope of the present work. I raise this point only to make it clear that these issues must also be considered by the interested reader, if the broad potential of FEL sources is to be fully appreciated.

The characteristics of single pass FEL's have been the subject of several sessions of this conference [1], representing a wide range of technological issues and approaches. As a basis for the present discussion, the Brookhaven National Laboratory Deep Ultra-Violet (DUV) FEL[2], and the Stanford Linear Accelerator Center Linear Coherent Light Source (LCLS) [3] are taken as possible sources operating in the UV and X-ray regions of the spectrum. Both operate as single pass devices, providing very high peak power and photon beam brightness. Since they are S-band 
linac driven machines requiring high peak currents for operation, the pulse repetition rates are unlikely to exceed $360 \mathrm{~Hz}$. Further, the output pulse widths range from $10 \mathrm{ps}$ to $10 \mathrm{fs}$, so these are comparatively low duty cycle sources. Although various options for multiple pulse formats with variable time delays and/or multi-color operation have been proposed, they are nominally single pulse sources i.e. without inherent micopulse structure. Both machines should provide flexible wavelength tuning over their specified output range. Beyond these common parameters, the machines and the research they engender begin to diverge.

The DUV FEL is a seeded beam amplifier. It relies upon 'conventional' laser technology for a 'seed' photon beam to couple with the electron beam. Through various arrangements, the FEL acts either to amplify at the same wavelength as the seed laser or, through harmonic generation and subsequent amplification, to frequency multiply and amplify the original laser pulse. The advantage of this approach is that the output radiation has the frequency stability and relative bandwidth of the seed laser. Electron beam brightness and energy variations are expressed in the output radiation only as changes in intensity. The pulse width and shape are also to first order determined by the properties of the seed laser. The obvious disadvantage of this approach is the short wavelength limit imposed by the seed laser and practical considerations for the construction of the accelerator driver.

To cross this threshold, the LCLS FEL takes advantage of the high electron beam energies available from the SLAC linac to drive a Self Amplified Spontaneous Emission (SASE) FEL. Because of the high energy from the accelerator, the demands on the wiggler are somewhat relaxed (long period and moderate peak field). A wiggler of significant length (the order of $40-70$ meters) is however required to achieve saturation at wavelengths less than $1 \mathrm{~nm}$. Since the LCLS is a SASE source, the output radiation wavelength stability depends upon the energy stabilization of the electron beam, and the intensity will be a strong function of the beam emittance and peak current. Clearly, for either source, the experiments must either be inherently insensitive to these inevitable variations, or suitable real-time, shot to shot diagnostics must be provided.

The foregoing discussion really contains all the essential information to determine what types of research are most suited to these machines. The operational wavelengths will determine the types of processes through which the source radiation will interact with the experiment. The tunability implies access to specific transitions, and opens a range of experimental opportunities as compared with line sources. The spectral properties (bandwidth, harmonic content, and source phase space) will be important in determining the requirements of the optical system for photon beam conditioning and transport. The high peak power is suggestive of studies of dilute systems, or non-linear optical studies where multi-photon excitations are important. The time structure of the radiation would provide a tool for the study of systems with picosecond dynamics, although the pulse separation would allow investigation of transient phenomena over much longer time 


\section{DISCLAIMER}

Portions of this document may be illegible in electronic image products. Images are produced from the best available original document. 
scales. These sources also possess a high degree of coherence (both spatial and temporal), thus interference effects might be effectively employed for imaging and intensity correlation studies. This collection of general attributes clearly suggests that these sources could be powerful research tools. What follows are a few examples of possible research problems which would be well served by sources of the type described above, with some closing comments about important issues for mounting experimental programs with these devices.

\section{Research in the Ultra-violet}

For a source operating in the vacuum ultraviolet, valence band electronic transitions are directly accessible by single photon excitation. This has important implications for the study of UV induced photochemistry, since the radiation is sufficiently energetic to break most chemical bonds. Molecules photoexcited by UV-VUV light frequently undergo fragmentation into atomic and/or smaller molecular species, most often radical species with open shells which readily undergo subsequent reactions with surrounding molecules and surfaces. To produce significant yields of these products the source must be extremely intense, providing pulse energies the order of $1 \mathrm{~mJ}$ per shot, and in nearly every case of even moderate complexity, the excitation wavelength will have a profound effect on the product distribution and yield. Operation at wavelengths shorter than $200 \mathrm{~nm}$ ( $\mathrm{LiF}$ window cutoff) allows the investigation of the electronic structure of many otherwise inaccessible species. This includes molecules such as $\mathrm{CH}_{4}, \mathrm{H}_{2} \mathrm{O}, \mathrm{CO}_{2}$, and most of the chloro-fluorohydrocarbons which are important in atmospheric photochemistry. The ability to perform the primary photochemistry by single photon excitation is a significant capability. One of the most powerful techniques for examining the state distribution of the reaction products involves so called vector correlation experiments, where the kinetic energy, momentum and identity of the photofragments are all determined. Much of the 'memory' of the primary photoexcitation event can be lost when multi-photon probes are utilized, as is often done with longer wavelength lab based lasers. In addition, the requirement for a well known and conveniently arranged electronic manifold to apply these multi-photon techniques can significantly limit the range of species available for study.

The benefits of an FEL source for this type of work are clear, the high peak power implies access to dilute systems which include gas phase samples, surface adsorbates, and transient species such as excited states or radicals. The tunability allows the exploration of resonant processes including excited states and threshold phenomena. The flexible time structure adds the dimension of time dependent phenomena, such as relaxation and excitation processes, and the possibility of coherent control of quantum dynamics in the time domain. For FELs which are driven (and in this case seeded) by 'conventional' lasers, synchronized multi-color pump-probe experiments become an obvious capability. 
Many of the winning features of the FEL source are equally applicable to other fields, such as biology . For example, the tunability of the source is of great importance in the study of radiation damage in DNA and other molecular targets in living cells. For wavelengths longer than $200 \mathrm{~nm}$, damage is induced by specific absorption and consequent photochemical changes in chromophores within the target molecule. At $\mathrm{x}$-ray wavelengths, the absorption occurs primarily in water, so damage mechanisms occur through a large number of indirect processes. The advantage of utilizing VUV radiation to elucidate damage mechanisms in DNA, proteins, membranes, and other biological targets, is that the energy-per-photon is just adequate to produce a limited number of ionizations. The number of chemical reactions produced in a VUV exposure is much more limited than in the case of $\mathrm{x}$-rays and other types of ionizing radiation, hence the complications resulting from a plethora of secondary reactions are avoided. Experiments of this type have been attempted utilizing synchrotron sources, but the comparatively low intensity available (in the requisite bandwidth) has, to date, restricted the range of experiments. To harvest a meaningful quantities of photo-products, doses must be measured in moles of photons.

Time resolved phosphorescence and fluorescence studies of biological systems could also benefit from an FEL source. In studies performed thus far, the samples are often far more concentrated than would occur in the native biological system. The quenching rates observed with these techniques are known to be extremely sensitive to the proximity of ions, other amino acid residues, and dependent upon the conformation of the molecule. This in fact forms the basis of the structural information derived from these techniques. A high peak power source, like the FEL, should allow this work to be performed in environments that are far more representative of real biological systems, so ambiguities due to intermolecular coupling of the target species can be avoided. The tunability of the source allows high resolution probing of specific sites within the molecule, while the flexible time structure provides the opportunity to optimize the data collection rate for the decay time and damage threshold of the specific molecule under investigation.

Similar advantages can be envisaged for many fields where one is interested in science related to the photo-excitation of comparatively low lying electronic transitions. Many additional experiments have been explored in chemical dynamics and biology beyond those briefly described above. Applications have also been developed in solid state physics, materials science, and atomic physics. Many. are predicated on extensions of existing research, where presently available sources have proved inadequate. While providing a semi-quantitative feel for the relative improvement made possible by an FEL, discussions of this type tend not to reach too far beyond existing science. This is much less the case for an X-ray FEL where the source is even further removed from existing technology. 


\section{Research in the X-ray region}

A source performing at the level of the proposed LCLS provides significant challenges to the imagination. It would produce a spectral brightness per shot which exceeds the brightness per second of third generation storage rings such as the ESRF, APS and Spring-8! With $150 \mathrm{fs}$ pulses, the LCLS would possess a peak brightness 10 orders of magnitude higher than the new sources. The wavelength range of the source from roughly 0.1 to $4 \mathrm{~nm}$, is suitable for examining core level electronic excitations, and perhaps more interestingly, is the appropriate length scale for a number of interesting imaging and scattering techniques. The tremendous coherence would be very useful for zone plate microscopy and holography in the water window ( 2 to $4 \mathrm{~nm}$ ), where single shot images could be obtained. At somewhat shorter wavelengths, 'normal' scattering techniques become viable although, given the pulse structure of the source, dynamics could be explored at previously unavailable time scales.

Considerable discussion has in fact be given to the subject of $\mathrm{x}$-ray time correlation spectroscopy (often known as speckle or intensity fluctuation spectroscopy) as a method to examine dynamics in condensed matter systems at time scales from $10^{-3}$ to $10^{3}$ seconds at length scales as small as $0.1 \mathrm{~nm}$. The technique depends upon the introduction of phase shifts from different domains of a specimen which will be observable as intensity fluctuations in the scattering. This is true only when the sample is illuminated with a sufficiently coherent source. A short wavelength FEL satisfies these requirements, and provides access to a much shorter length scale than is available from any existing source.

A number of problems have been described where the understanding of microscopic dynamics could be enhanced through the use of time correlation spectroscopy. Among these are free standing smectic liquid crystal films, dipolar glasses, and thin film magnetic materials; all of potentially great technological importance. The source brightness would allow single shot speckle patterns to be obtained, and in principle, the time structure of the source would allow dynamics to be studied to the limit of the FEL repetition rate. Another side benefit of the source brightness is the possibility of examining small samples using a variety of otherwise 'conventional' $\mathrm{x}$-ray techniques. This is simply a result of the small source divergence .

Reaching a bit further beyond the present state of the art, interferometric techniques of various kinds have also been suggested, which again exploit the unique attributes of an x-ray FEL. For example, interference patterns on a sub-nanometer scale could be generated either to characterize or manipulate materials. Coherent lattice motions might be induced to drive specific structural rearrangements. By extension, the source tunability and polarization could be employed to resonantly enhance a particular process or induce a magnetic periodicity. The decay of these prepared states could be used as a characterization technique. Utilizing the FEL in this fashion could lead to a new suite of probes for studying the dynamics of both structural and 
magnetic order at short length scales. This could be especially valuable for the study of incommensurate systems.

The source brightness should also be sufficient to mount serious experiments on the nonlinear optical properties of materials in the x-ray regime. Multi-color experiments could be envisioned where optically induced charge density modifications (say from a UV laser source) are probed with a pulse from an x-ray FEL. Alternatively, the FEL pulse could be split and time delayed to serve as the source for both the pump and probe beam. Multiple color experiments might also be possible using either harmonics from the FEL, or the continuum background, if they provide adequate intensity for the particular experiment being conducted.

Clearly, $\mathrm{x}$-ray FELs provide a host of exciting attributes, many of which are unavailable individually in any other source, and certainly not as a collective capability. Divining the new science that machines of this type engender is a tremendously challenging task, since the properties of the source are so far beyond current capabilities. Proposals for FELs of this type are sufficiently new to the research community, that I have no fear in asserting that the most exciting applications for this source are yet to be described.

\section{Closure}

This brief look at potential research applications for short wavelength FEL amplifiers is perhaps less indicative of the research opportunities than it is of the sociology which will likely lead to their successful development and utilization. The UV FEL based science is close enough, in some respects, to existing laser derived technology that it borrows much from the communities that use conventional lasers for their research. Among these groups, the chemical and atomic physics communities are perhaps the most accustomed to being involved in the development of the photon sources for their research, the so-called 'laser jocks' of the world. These communities were the first to seriously examine the potential of UV FELs on their science. Involvement from the biological sciences has been somewhat slower in developing, largely because photon sources are regarded as important tools for research; not the subject of research for this community. These machines could never-the-less become an important resource for work conducted in biology and other fields of research that are not, as yet, part of the 'scientific case' for short wavelength FELs. 
FELs are accelerator based sources, and much of the early application development for short wavelength FELs is now taking place at synchrotron sources. This is in part because the psychology of going to a remote location to perform experiments is familiar to synchrotron radiation researchers. A major component of this community is involved in condensed matter research, so it is not at all surprising that the problems which have been suggested for $\mathrm{x}$-ray FELs are largely derived from that community. The difficulty is that even third generation source are, in many instances, inadequate to the task of providing a platform even for proof of principle experiments of the type which could be mounted at an FEL. They might be suggestive of interesting science, but the foibles of FELs must be considered along with their potential experimental benefits.

For example, experiments performed at synchrotrons have a comparatively easy task in intensity normalization as contrasted with FELs. The experiments are usually only linear in nature (spectroscopies for example), and intensities at storage rings vary comparatively slowly. By contrast, at an FEL, many experiments may be inherently non-linear in nature (thus more sensitive to intensity variations), and the source variation shot-to-shot could be significant. A new problem for synchrotron people, old news to the laser folks. Similarly, refractive optics are clearly impractical at wavelengths shorter than $200 \mathrm{~nm}$, which leaves laser people on unfamiliar ground, but practitioners of UV science at synchrotrons have long ago come to grips with this problem.

Clearly, what is required for the growth of short wavelength FEL research, is a fusion of approaches and ideas from practitioners of laser based research, and researchers who presently work at synchrotron sources. Further, source and science development need to advance together, so it is important to foster interaction between the machine 'builders' and 'users' at an early stage. This is one of many instances where I feel that the sum of the efforts of a coordinated, communicative community will vastly outstrip the performance of its individual members when left to their own devices.

\section{DISCLAIMER}

This report was prepared as an account of work sponsored by an agency of the United States Government. Neither the United States Government nor any agency thereof, nor any of their employees, makes any warranty, express or implied, or assumes any legal liability or responsibility for the accuracy, completeness, or usefulness of any information, apparatus, product, or process disclosed, or represents that its use would not infringe privately owned rights. Reference herein to any specific commercial product, process, or service by trade name, trademark, manufacturer, or otherwise does not necessarily constitute or imply its endorsement, recommendation, or favoring by the United States Government or any agency thereof. The views and opinions of authors expressed herein do not necessarily state or reflect those of the United States Government or any agency thereof. 


\section{Acknowledgments}

This work was performed under the auspices of the United States Department of Energy, under contract DE-AC02-76CH00016.

\section{References}

$116^{\text {th }}$ International Free Electron Laser Conference, these proceedings. See for example papers from sessions on Single Pass UV/X-ray Free Electron Lasers, and Prebunched Beams and Coherence.

2 The Brookhaven National Laboratory Deep Ultra-Violet Free Electron Laser Conceptual Design Report (2 Volumes) January 1994, BNL Publication Number 49713

3 Workshop on Scientific Applications of Coherent X-rays, Proceedings from Workshop held February 12 1994, SLAC Publication 437, SLAC/SSRL-0066.

(n.b. Word count 3145) 